\title{
CAMEROUN : DE L'ENCOURAGEMENT DES NAISSANCES \\ A LA MAITRISE DE LA FECONDITE \\ CAUSES ET CONSEQUENCES DE L'EVOLUTION \\ OBSERVEE
}

Nicole BELLA

\section{Introduction}

Le Cameroun, comme la plupart des pays d'Afrique noire, connaît une situation démographique qui, sans être pour le moment dramatique, n'en inquiète pas moins les autorités gouvernementales de ce pays. En effet, le taux d'accroissement naturel y était estimé à 2,92\% en moyenne par an en 1987 (résultat provisoire du 2ème recensement de la population, qui a eu lieu à cette date). Cette forte croissance démographique est le résultat d'une natalité élevée et constante (43,4 pour mille en 1987) et d'une mortalité plus faible et en baisse (14 pour mille en 1987). Le taux d'accroissement a connu une évolution vers la hausse puisqu'il était de 2,2\% dans les années 60 . Cette évolution risque de se poursuivre si les tendances actuelles de la mortalité et de la natalité se maintiennent.

La croissance de la population n'est cependant pas remise en cause en tant que telle. En effet, une certaine augmentation du volume de la population est souhaitable, dans la mesure où elle peut contribuer positivement au développement économique et social du pays et ralentir le vieillissement. Le problème qui se pose concerne la vitesse de cet accroissement qui risque de compromettre l'équilibre entre la population et les ressources. En effet, compte tenu des ressources disponibles et des graves problèmes économiques auxquels il est actuellement confronté, le Cameroun, selon les autorités gouvernementales, ne peut pas se permettre de voir sa population doubler tous les 24 ans (compte tenu du taux d'accroissement actuel). C'est ainsi qu'ont été prises dès 1980, des mesures visant à ralentir la croissance démographique et à réduire ses effets négatifs sur le développement socio-économique du pays, l'environnement, le bienêtre familial, etc. L'heure est donc actuellement, au Cameroun, au contrôle de la dynamique de la population. Une phrase extraite du 
discours du Président de la République Paul BIYA en 1986 à l'Assemblée nationale, à l'occasion de la présentation du 6ème plan quinquennal de développement, en dit long sur la nouvelle position du gouvernement camerounais quant aux problèmes démographiques :"...La procréation fût-elle un droit fondamental de tout homme, peut et doit être maîtrisée...". Il y a encore une quinzaine d'années, on ne pouvait concevoir une telle opinion au Cameroun. C'est dire çue depuis 1974 , date à laquelle a eu lieu la conférence mondiale sur la population à Bucarest, beaucoup de choses se sont passées dans ce pays, comme en Afrique noire en général.

A l'époque, et surtout avant cette date (c'est-à-dire 1974), la position la plus communément admise était que la population était insuffisante tant pour les besoins du développement économique que pour le désir de grandeur nationale. Officiellement le Cameroun ne souffrait pas de problème démographique, exception faite de l'inégale répartition spatiale de la population et de l'exode rural. Il fallait donc encourager l'accroissement de la population.

Le passage d'une vision nataliste à un désir de maîtrise de la croissance démographique a donc été lent, et la décision de mettre en oeuvre une politique de fécondité a été tardive. Les problèmes posés ou pouvant être posés par une croissance rapide de la population $(2,2 \%$ en moyenne par an dans les années 60 , selon l'estimation du Ministère du Plan et de l'Aménagement du Territoire) ayant déjà été entrevus dès 1966, cette décision aurait pu être prise en effet par le gouvernement dès ce moment, ce qui aurait permis de limiter les inconvénients de cet accroissement.

Nous traiterons dans cet article d'une part, des raisons qui pourraient expliquer l'hésitation des autorités du Cameroun à se départir de leur natalisme avant 1980 : c'est ce que nous appelons les causes du blocage ; des facteurs qui ont pu permettre le changement observé depuis 1980, (facteurs de déblocage) et enfin des conséquences de cette nouvelle orientation : les effets induits. Cependant avant d'entrer dans le vif du sujet, il est nécessaire de faire un historique de la position du gouvernement camerounais quant à la population. 


\section{Historique}

Depuis 1980, le Cameroun ne fait plus partie des pays qui encouragent leur natalité. D'un natalisme affiché, ce pays a évolué vers une prise en considération des conséquences de son accroissement démographique, estimé à environ 2,5\% par an par le recensement de 1976. Cette croissance qui inquiète aujourd'hui, a pourtant été longtemps souhaitée.

En effet avant 1980, la population en tant que facteur de développement et atout politique était valorisée. Au lendemain des indépendances, les pays africains, dont le Cameroun, dans leur désir de consolider leur souveraineté nouvellement acquise (le Cameroun a obtenu son indépendance le ler Janvier 1960), tiraient quelque fierté à avoir une population nombreuse, non seulement pour des raisons de grandeur nationale, mais aussi et surtout pour des besoins de développement économique. La croissance de la population n'était par conséquent pas considérée comme un problème, au contraire, il fallait l'encourager, le marché étant jugé insuffisant pour assurer l'écoulement des produits. En effet, dans le rapport de politique générale du chef de l'Etat, le 25 Septembre 1960, il est dit:" parmi les limitations à l'incitation à investir, il faut citer les facteurs économiques tels que la faible densité du marché local, situé en dessous du seuil de rentabilité (...)".

La population du Cameroun, estimée à l'époque à quatre millions d'habitants, était largement en deçà de ce seuil de rentabilité, fixé à dix millions de consommateurs. Il semblerait donc que, selon les autorités gouvernementales, l'effectif limité de la population ne permettait pas de réaliser de façon adéquate deux objectifs : d'une part, disposer d'un marché économique suffisant pour rentabiliser les investissements, d'autre part, sortir le pays du sous-développement. Le chiffre modéré de la population ne permettait pas non plus, aux dirigeants du Cameroun de satisfaire leur désir de grandeur nationale, comme en témoigne $\mathbf{M}$. Ahmadou AHIDJO dans son discours du 21 Décembre 1964 : "Moi aussi, j'arrive à rêver que mon pays est aussi grand que les Etats-Unis d'Amérique et aussi grand que la Russie. Mais quand je me réveille, je constate que mon pays c'est le Cameroun avec ses quatre millions d'habitants". 
Ceci explique pourquoi les naissances étaient encouragées. Cependant la venue des enfants pouvant alourdir les charges des familles, une législation de protection familiale a été prévue. Le Cameroun pour traduire dans les faits son discours nataliste, adopta des moyens d'action à la fois directs et indirects. D'une part la législation sur l'avortement interdit toute interruption de grossesse, à l'exception de l'avortement thérapeutique ou médical (article 339 du Code pénal), à condition toutefois que ce dernier soit accompli par une personne habilitée (c'est-à-dire un médecin) et pour deux raisons bien précises : sauvegarder la vie de la mère en danger, ou alors en cas de viol.

D'autre part, jusqu'en 1980, la vente des contraceptifs au public et la publicité anticonceptionnelle étaient interdites, par la loi $n^{\circ} 29 / 69$ du 29 Mai 1969, portant réglementation de la profession de pharmacien au Cameroun. Ces deux législations sont en fait un héritage de la colonisation. La loi interdisant la contraception au Cameroun étant issue de la loi française de 1920.

Outre ces moyens directs, d'autres tendaient de façon indirecte, à travers notamment la politique des revenus, à agir sur la population, à savoir d'une part les prestations familiales (instituées par l'article 1er de la loi 67-LF-7 du 12 Juin 1967) accordées aux seuls salariés du public et du privé, d'autre part, le système d'imposition, qui comprend:

la taxe professionnelle, laquelle frappe à des taux différents les bénéfices et revenus des particuliers,

- $\quad$ la surtaxe progressive, en fait l'impôt sur le revenu net global, à caractère personnel. Ce dernier, dû par les salariés, est retenu mensuellement par leur employeur. Son montant est inversement proportionnel au revenu du salarié et au nombre de ses enfants à sa charge. Cependant, depuis 1969, le bénéfice du dégrèvement fiscal est limité à 7 enfants.

La politique des revenus en l'occurrence les prestations familiales, est cependant limitée aux seuls salariés et exclut la frange la plus importante de la population, à savoir les paysans (72\% de la population totale, selon le recensement de 1976) qui ont davantage d'enfants $(6,1$ enfants en moyenne par femme selon l'Enquête 
Nationale de Fécondité de 1978, contre 5,3 en milieu urbain). En outre, leur montant est pour le moins modique. Par exemple, l'allocation familiale attribuée à tout enfant à charge de moins de 21 ans, quel que soit son rang, s'élevait à 1500 FCFA (soit 30 FF) par mois en 1978.

Par conséquent, si l'intention de favoriser la croissance démographique apparaît dans le discours gouvernemental à cette époque (1960-1980), les moyens pour y parvenir semblent plutôt limités, du moins en ce qui concerne la politique des revenus, qui semble être davantage une politique de protection et de justice sociale (cependant limitée à une petite partie de la population) qu'une politique démographique. Les mesures mentionnées ci-dessus sont donc plus ou moins le reflet d'un discours gouvernemental nataliste, qui va cependant connaître un point de rupture dès 1980 .

En effet comme nous l'avons dit plus haut, le gouvernement, conscient de l'incidence de l'accroissement démographique sur le développement économique et social, décide en 1980 de maîtriser la croissance de sa population. Dans un discours de politique générale tenu le 13 Février 1980 à Bafoussam ${ }^{1}$ l'ex-Président de la République, Mr. Ahmadou AHIDJO évoque : "le spectre préoccupant de l'explosion démographique caractéristique des pays en développement". Explosion, poursuit-il, "dont nous devons dès maintenant tenir le plus grand compte en vue de la maîtrise de notre développement au cours des années à venir (...)". Comme solution à ce problème démographique, le gouvernement camerounais préconise la parenté responsable. Il s'agit pour chaque Camerounais de prendre conscience de ses responsabilités et de choisir librement, en toute connaissance de cause, le nombre d'enfants qu'il désire, selon ses moyens et le niveau de vie auquel il aspire. Le gouvernement se propose de susciter cette prise de conscience en sensibilisant les familles sur les problèmes posés par une forte croissance démographique, aussi bien sur le plan national qu'au niveau du bien-être familial. Un projet de constitution d'une Commission Nationale de la Population (CNP) est mis à l'étude. Cette instance sera chargée de définir une politique de population, dans le but de maîtriser l'accroissement de la population. La nouvelle position du gouvernement camerounais, quant aux questions démographiques (réaffirmée et poursuivie par Mr. Paul BIYA qui a succédé à Mŕ. 
AHIDJO en 1982) sera rendue officielle, lors de la conférence mondiale de la population de Mexico, en Août 1984. Intervenant dans le débat général, la représentante du Cameroun, Mme Elizabeth TANKEU (à l'époque vice-ministre du Plan et de l'Aménagement du Territoire), affirme : "...Conscients de l'incidence de l'accroissement de la population sur les objectifs du développement, nous n'encourageons pas la prolifération des familles nombreuses et nécessiteuses. Nous pensons orienter notre politique démographique vers l'amélioration de la qualité de la population...". Ceci se fera entre autre par le biais de la planification familiale, qui signifie au Cameroun:

espacement et non limitation des naissances, pour sauvegarder la santé de la mère et de l'enfant et pour promouvoir la parenté responsable ;

lutte contre la stérilité et l'infécondité, en informant notamment les individus, sur les maladies sexuellement transmissibles.

Cependant, si le désir de contrôler l'accroissement démographique a été réaffirmé à Mexico, certains dirigeants politiques camerounais n'en pensent pas moins, que cet accroissement n'a pas que des effets défavorables sur le développement. Ainsi Mme E. TANKEU, après la conférence de Mexico, a déclaré au quotidien Cameroun Tribune (du 24/9/1984): "Dans la mesure où la population est pour nous une ressource, avant d'être une charge, nous pouvons dire que les 14 millions d'habitants qu'abritera le Cameroun en l'an 2000, constituent un atout certain, pour la valorisation d'importantes potentialités économiques que recèle notre pays. Le gouvernement, quant à lui, s'efforce, dans le cadre de notre système de planification, de préparer un cadre de vie meilleur à cette population en expansion".

Les arguments qui justifiaient avant 1980 la politique nataliste du gouvernement, sont donc remis à l'ordre du jour. La position nuancée du Cameroun, telle qu'elle apparaît à Mexico, indique que le gouvernement de ce pays, bien qu'ayant conscience des problèmes posés par une croissance rapide de la population et désirant y apporter un remède, entretient une ambiguité quant au volume de sa population (estimée à 10,5 millions d'habitants par le recensement de 1987). Il 
pensait encore à l'époque, que le pays était sous-peuplé. Ce dernier point, couplé avec le contexte socio-culturel du pays, justifiait, selon lui, ses arguments natalistes.

\section{Les causes du blocage jusqu'en 1980}

\section{Les raisons politiques}

Les problèmes posés par une croissance rapide de la population ayant été entrevus dès 1966, nous pensons que les causes qui expliquent qu'une décision n'ait pas été prise à cette époque, pour ralentir cette croissance sont pour la plupart politiques. Politiques, car utilisées comme telles par les autorités gouvernementales, pour justifier leur natalisme. Il était en effet communément admis que le pays était souspeuplé, ce qui ne favorisait pas le désir de grandeur nationale et le développement économique du pays. N'était-il pas dit dans le Ilème plan de développement (1966-1971) que "le nombre et la qualité des hommes qui forment une nation constituent évidemment l'un des facteurs essentiels de cette nation"?

Le rôle de la population dans le développement est très souvent avancé par les natalistes, selon lesquels un taux de croissance démographique élevé peut accroître "les facultés d'adaptation d'une économie et augmenter par conséquent l'aptitude de celle-ci à répondre aux exigences du progrès économique"2.

Ce rôle de la dynamique de la population comme facteur de développement, va de pair avec une confiance parfois exagérée, dans les importantes ressources naturelles, encore inexploitées, dont disposerait le pays. Mais cette dynamique démographique n'est efficace que jusqu'à un certain point, c'est-à-dire quand la croissance économique peut suivre celle de la population. En effet, s'il est vrai qu'une forte population permet: de disposer d'une main-d'oeuvre suffisante pour exploiter des ressources, d'élargir le marché nécessaire pour absorber et rentabiliser une production de masse, de réaliser des économies d'échelle, et par exemple en faisant pression sur les terres disponibles, de recourir à des techniques de haut rendement, il est vrai aussi qu'un taux de croissancẻ démographique élevé peut être un obstacle au développement, dans ce sens que les investissements 
démographiques peuvent freiner les efforts nécessaires, pour élever le niveau de vie de toute la population ${ }^{3}$. L'augmentation du nombre d'habitants gonfle peut-être les besoins et accroît la consommation. Malheureusement ou heureusement, l'économie du Cameroun n'est pas fermée. Les Camerounais sont de plus en plus amenés à consommer des produits importés, soit parce qu'ils coûtent moins cher, soit parce que la production intérieure est insuffisante, soit pour certains, pour des raisons de prestige. On voit mal, dans ce cas, comment une population importante en nombre peut accroître la demande ou la consommation.

Si la croyance en l'existence du sous-peuplement du pays pouvait s'expliquer à l'époque (les années 60 ) par la non disponibilité de données démographiques fiables, il est pour le moins curieux que cet argument soit encore avancé actuellement, alors que la population est mieux connue. Il en va de même des conséquences favorables de la croissance démographique sur le développement, sur lesquelles on continue à mettre l'accent.

La mise en place d'une politique de maittrise de la croissance démographique a été décidée par l'ex-Président Mr. Ahmadou AHIDJO en 1980. Cependant, cette politique se révèle être un compromis, entre le désir de tenir compte de l'équilibre entre population et ressources et celui, non moins évident, de ne pas heurter les convictions des natalistes. Natalistes que l'on trouve aussi bien au sein du gouvernement, qu'au sein du parti RDPC (Rassemblement Démocratique du Peuple Camerounais). Ce dernier, bien que devant, en principe soutenir toute action du gouvernement, dont celles relatives à la politique de population, reste fondamentalement nataliste. Il existe ainsi un décalage entre la volonté affichée du chef de l'Etat, également président du parti, de maîtriser la croissance de la population, et le parti, qui tout en le soutenant, tient à marquer sa position. Celle-ci a été exprimée par un responsable du RDPC, dans le cadre du séminaire de sensibilisation des journalistes africains aux questions de population, tenu à Yaoundé en Mai 1989. Sa communication dont nous allons faire ressortir ci-dessous les principaux points, a fait l'effet d'une fausse note dans ce séminaire portant sur le contrôle de l'accroissement démographique. 
Le RDPC justifie notamment sa position nataliste en affirmant que le Cameroun, compte tenu de l'étendue de son territoire et de ses ressources naturelles, peut avoir une population supérieure à son effectif actuel. Un certain nombre d'arguments viennent étayer ces déclarations :

- $\quad$ Les pays sous-développés sont sous-peuplés, tandis que les pays développés sont dans l'ensemble, ou surpeuplés, ou proches de leur seuil optimum de population. La conclusion logique est qu'il faut accroître la population pour développer le pays.

- Le taux de progression des populations du Tiers-monde en général, et du Cameroun en particulier (un peu plus de 3\% en moyenne par an) ouvre de larges espoirs dans ce sens (développement).

- D'après la Banque Mondiale, la population camerounaise peut croître jusqu'à 51 millions d'habitants. Donc notre pays se meut et demeurera longtemps encore avec un large déficit de population.

Ceci montre que l'on continue à raisonner en Afrique et au Cameroun en particulier, en termes de volume de la population. Or ce n'est pas tant la quantité de la population, que son rythme d'accroissement qui importe.

Les fortes masses, comme les fortes densités de population, n'empêchent ni ne freinent le développement, dans les pays d'Europe, au Japon ou dans les pays moyennement avancés comme la Corée du sud, etc.

Nos auteurs oublient que ces pays ne sont pas forcément développés parce que surpeuplés, mais parce qu'ils ont su mettre en place des économies capables de faire vivre leurs populations, ce qui n'est pas le cas de la plupart des pays en développement.

Le RDPC est donc convaincu que l'encouragement du peuplement du Cameroun est un impératif politique, économique et stratégique. Selon lui, "il serait en effet difficile, voire impossible, de 
développer convenablement ce pays, de garantir convenablement son indépendance et son intégrité territoriale, d'assurer son progrès et son bonheur constant dans tous les domaines, sans une population suffisamment abondante, pour être en permanence à même de trouver en son sein, des hommes et des femmes capables d'imaginer et de réaliser son destin".

Les arguments des natalistes sont souvent fondés sur quelques exemples historiques, le plus souvent tirés de l'Occident, où la croissance démographique a pu jouer un rôle dans le développement économique de certains pays, jusqu'au point où la population devenait un obstacle au développement, d'où le recours à l'émigration (EtatsUnis, Canada, Australie, etc). Les pays en développement quant à eux, ne disposent pas de cet ultime recours.

Population et développement sont liés, mais selon J-C CHESNAIS,"il est malaisé de prévoir à priori le rôle de l'accroissement démographique dans le développement économique, tant sont multiples les circonstances et les paramètres en jeu"4. Les raisons politiques qui expliquent, selon nous, le retard pris par le gouvernement pour mettre en place une politique de maîtrise de la croissance démographique, sont essentielles, mais elles ne sont pas les seules. D'autres ont pu jouer.

\section{Les raisons démographiques : la stérilité}

Le sous-peuplement du Cameroun, résulte aussi bien d'une mauvaise répartition de la population sur le territoire, que du niveau de stérilité que connaît le pays (ces deux choses étant bien sûr liées).

Facteur limitant la fécondité au niveau individuel, la stérilité est toutefois un argument utilisé par le gouvernement, pour justifier sa politique nataliste, en ce sens qu'il encourage la croissance de la population, pour peupler les espaces vides, et donc équilibrer la population sur l'ensemble du territoire.

Le Cameroun où subsiste une stérilité élevée, malgré une baisse enregistrée depuis les années 1960 (baisse qui n'a cependant pas touché de la même façon toutes les régions, dont le nord du pav's $\mathbf{s}^{5}$ ), n'est pas 
un cas isolé. Il fait en effet partie d'une ceinture de sous-fécondité et de stérilité qui concerne la quasi totalité des pays d'Afrique subsaharienne, principalement ceux d'Afrique Centrale, dont le Gabon, le Congo, la République Centrafricaine ${ }^{6}$. On ne dispose pas de chiffres récents sur ce phénomène. Les seules données existantes sont celles de l'Enquête Nationale de Fécondité (ENF) de 1978 (voir en annexe tableau 2). Selon cette enquête, $22 \%$ des Camerounaises âgées de 15 à 54 ans seraient stériles ou infécondes. Cette proportion regroupe d'après le tableau 2 aussi bien les femmes n'ayant jamais été enceintes (10\%) que les femmes sans grossesse féconde depuis plus de 5 ans $(12 \%)$.

La stérilité varie selon l'âge des femmes. Elle frappe plus les groupes d'âge avancé que les jeunes (hormis les 15-19 ans, pour lesquelles l'infécondité observée traduit le fait qu'elles sont encore en début de vie féconde). Ceci peut être aussi bien un effet d'âge, qu'un effet de génération. La lutte engagée contre la stérilité depuis les années 60 profiterait plus aux jeunes générations.

Le taux de stérilité observé au niveau national varie selon les régions (annexe : tableau 3). Les régions les plus touchées (véritables poches de stérilité et de sous-fécondité) sont celles du nord du pays (18\% de femmes en union depuis 5 ans sans grossesse féconde), le centre-sud (16\%), l'est (12\%). Les moins affectées sont l'ouest du pays $(5 \%)$, le sud-ouest $(3 \%)$. Ces dernières régions sont également celles où l'on enregistre le nombre moyen d'enfants le plus élevé (annexe : tableau 4) : Ouest (7,7 enfants par femme, alors que la moyenne nationale est de 6,5), sud-ouest $(7,6)$. Les niveaux de stérilité et de fécondité sont donc inversement proportionnels.

Les causes de cette stérilité n'ont pas totalement été mises en lumière. Cependant, on estime que la majorité des cas de stérilité (surtout la stérilité secondaire) sont provoqués par les maladies sexuellement transmissibles. D'ailleurs la lutte contre ces dernières (introduction de la pénicilline) a pu faire reculer le niveau de stérilité. Mais, en dépit des progrès accomplis, ce phénomène reste un problème de santé publique au Cameroun, d'où la volonté du gouvernement, qui y a vu dans le temps une raison pour encourager l'augmentation de la population, d'y remédier. La maîtrise de la fécondité, aujourd'hui 
prônée, doit passer par une réduction de la stérilité. Mais n'est-ce pas là un paradoxe ? En effet, comment peut-on vouloir en même temps réduire la fécondité et lutter contre la stérilité, alors qu'on sait qu'une diminution de celle-ci entraînerait forcément une élévation du niveau de fécondité, comme cela a été le cas au Cameroun entre 1965 et 1978 ? L'indice de fécondité était en effet passé de 5,4 enfants en moyenne par femme au cours de la période 1960-1965, à 6,5 en 1978 (ENF) (voir annexe). La contradiction n'est en fait qu'apparente, car s'il est vrai qu'une baisse de la stérilité entraîne à court, voire à moyen terme, une hausse des taux de fécondité, il est tout aussi vrai qu'un niveau de stérilité élevé peut favoriser la fécondité en ce sens qu'il aboutit à l'amplification de la procréation de chaque femme féconde. Dans un contexte culturel où l'enfant, et encore plus la famille nombreuse, sont hautement valorisés, la stérilité, dont les conséquences sont multiples pour l'individu et surtout pour la femme, est vécue comme un drame et perçue comme un non accomplissement de soi ${ }^{7}$. Un proverbe Yoruba traduit très bien cette situation : "Les enfants sont les habits du corps; sans eux vous êtes nus". Dans un tel contexte donc, toute femme doit se valoriser en faisant la preuve de sa fécondité. La valeur d'une femme se mesurant au nombre d'enfants qu'elle met au monde, les femmes fécondes, qui s'estiment avantagées par rapport à celles qui sont stériles, tendraient donc à maximiser leur fécondité. Il en découle qu'une politique nationale de maîtrise de la fécondité ne peut se concevoir sans qu'un remède soit apporté à la stérilité qui est une absence de choix. En effet, il est nécessaire de donner à toutes les femmes une chance d'avoir des enfants afin qu'elles puissent choisir en toute conscience et en toute liberté leur nombre et le moment où elles les voudront.

\section{Les raisons culturelles}

Le contexte culturel pourrait expliquer l'hésitation ou le retard du gouvernement, à mettre en place une politique de maîtrise de croissance démographique, (d'autant plus que ce dernier s'y est référé ou s'y réfère encore, pour justifier d'une part son prognathisme, d'autre part son souhait en ce qui concerne le ralentissement du taux d'accroissement naturel), ainsi que le fait de ne pas imposer de façon autoritaire aux Camerounais une dimension familiale spécifique. 
Ce contexte culturel au Cameroun se caractérise par une demande très forte d'enfants. L'ENF (1978) avait révélé que les Camerounaises désiraient avoir 8 enfants en moyenne par femme. Ce désir va de pair avec une pratique faible de la contraception, laquelle, peut s'expliquer par une offre insuffisante de moyens contraceptifs, mais résulte surtout d'une demande limitée, voire quasi inexistante de ces méthodes. Pourquoi, en effet, recourir à la contraception si l'on désire avoir une nombreuse progéniture? Il en découle que la contraception n'est actuellement pratiquée que par moins de $5 \%$ des femmes camerounaises, surtout jeunes (20-30 ans), habitant Yaoundé et Douala, les deux villes les plus importantes du pays. Cette estimation est confirmée par les résultats provisoires de l'Enquête démographique et de Santé réalisée au Cameroun en 1991.

La forte demande d'enfants est conditionnée par la valorisation de la fécondité dans la société ${ }^{8}$ Dans cet univers resté marqué par l'animisme, et où les religions traditionnelles continuent d'être pratiquées, malgré la christianisation et l'islamisation, l'enfant confère un statut important. Signe de richesse, de prospérité sociale, l'enfant confere respect. Il n'est pas seulement un "bien", il est aussi source de richesse. Dans une société comme celle du Cameroun, avoir beaucoup d'enfants signifie davantage de revenus pour les familles pauvres, et aussi une aide matérielle supplémentaire pour les activités agricoles par exemple.

Une nombreuse progéniture est également considérée comme une bénédiction des dieux et des ancêtres. Avoir ou non des enfants ne dépend par conséquent pas de la volonté des individus, mais de celle d'un être suprême. Ces croyances conduisent très souvent, à dégager les individus de toute responsabilité dans leurs comportements procréateurs, et donc ne les incitent pas à décider eux-mêmes de la taille de leurs familles. De telles croyances vont de pair avec un niveau d'instruction plutôt bas. Mais ceci ne signifie pas, que la forte demande d'enfants ne serait que le fait des catégories de la population peu ou pas instruites. En effet, au Cameroun, même une certaine élite, pourtant plus en contact avec les pratiques modernes et occidentales, reste aussi fortement attachée à la tradition. Le besoin d'enfants ici, bien que relativement inférieur par rapport aux catégories moins instruites, reste toutefois élevé (les femmes mariées ayant fait des études secondaires 
ou supérieures désiraient en moyenne 6,4 enfants selon ENF contre 8,2 pour les femmes n'ayant jamais été à l'école). Il s'explique plus par des raisons de perpétuation de la lignée, que par le désir de respecter la volonté d'un être suprême, ou le désir d'un statut social que cette élite a acquis par d'autres moyens que par les enfants.

La demande d'enfants des femmes camerounaises est conditionnée aussi et surtout, par les désirs de leurs maris. Si les femmes ont pu intérioriser la valeur accordée à la fécondité, qui leur confère l'un des seuls statuts qui leur est reconnu, force est de constater qu'en Afrique et au Cameroun en particulier, la volonté de l'homme prime dans le couple. L'homme étant en général le garant de la tradition, celui qui doit perpétuer la famille, son option détermine largement la demande effective et même la fécondité réalisée du couple 9 . Ainsi lorsqu'une femme, pour une raison ou pour une autre, désire arrêter temporairement ou définitivement les naissances, elle ne peut légalement le faire sans le consentement de son mari.

Le besoin d'enfants au Cameroun va de pair avec la solidarité communautaire. Les enfants n'étant pas uniquement ceux du couple ou de l'individu qui les a mis au monde, ils sont très souvent confiés à d'autres membres de la famille. Cette solidarité, à bien des égards nécessaire, dans la mesure où elle allège le poids des charges familiales parfois lourdes, peut favoriser la non-responsabilité parentale et inciter les individus à procréer.

En effet, la responsabilité parentale (en termes d'éducation et d'entretien des enfants) n'étant pas toujours ou vraiment assurée par les géniteurs eux-mêmes, les individus peuvent faire autant d'enfants qu'ils le désirent, puisque de toutes manières, ils peuvent toujours se retourner vers la famille élargie pour se décharger de leur responsabilité parentale.

Ces raisons socio-culturelles, malgré le coût de plus en plus élevé de l'éducation des enfants, et une légère évolution des mentalités, due notamment à l'élévation du niveau d'instruction et de vie (qui fait apparaître d'autres moyens que la procréation pour satisfaire les besoins et aspirations fondamentaux), continuent à déterminer fortement la fécondité. La procréation restant une valeur en soi, il s'avère difficile 
de prévoir une baisse de la fécondité, laquelle baisse, si elle se produit, sera due à d'autres paramètres, tels que le souci des femmes de sauvegarder leur santé et celle de leurs enfants. Les pouvoirs publics ne s'y sont pas trompés, qui prônent l'espacement des naissances comme méthode de planification famiiiale, devant aboutir à un ralentissement de la croissance démographique.

En conclusion de cette première partie, soulignons que les différentes raisons évoquées qui ont pu à des degrés divers, expliquer les hésitations ou le retard dans la mise en place d'une politique de population, ne sont pas indépendantes les unes des autres. Les problèmes liés à la stérilité, qui font que les individus cherchent à maximiser leur fécondité, ne peuvent se comprendre que par rapport au contexte socio-culturel nataliste, qui "pénalise", en quelque sorte, les personnes stériles ou sans enfants vivants.

En 1980, la situation de blocage observée jusqu'alors se dénoue. En effet, dans un discours au congrès de Bafoussam, le chef de l'Etat en exercice à l'époque, M. Amadou Ahidjo, face à une situation démographique qu'il considère comme explosive, décide la mise en oeuvre d'une politique de maîtrise de la croissance de la population jugée trop rapide. Un certain nombre de facteurs expliquent ce déblocage. Seront par conséquent traités, dans la troisième partie de cet article, les effets moteurs du changement de perception du gouvernement camerounais.

\section{Les facteurs de deblocage ou effets moteurs du changement}

En 1980, les autorités gouvernementales dont la ligne politique, en matière démographique était jusqu'alors nataliste, décident de changer de cap. Si les raisons de ce changement, comme le dit Patrick Gubry ${ }^{10}$, restent en partie obscures, il n'en demeure pas moins que certains faits ou facteurs l'ont rendu possible. 
1. Le rôle des deux conférences mondiales sur la population (Bucarest, 1974 et Mexico, 1984) et plus spécifiquement du Plan d'Action Mondial adopté à Bucarest

La conférence mondiale sur la population organisée par les Nations Unies à Bucarest (Roumanie) du 19 au 30 août 1974 avait pour but d'examiner les problèmes démographiques fondamentaux, leurs liens avec le développement économique et social, et les politiques et programmes démographiques nécessaires pour promouvoir le bien-être et le progrès ${ }^{11}$. Malgré ces thèmes éminemment techniques, c'est la politique, mieux, l'idéologie, qui domina ces débats.

La Conférence de Bucarest a lieu, à un moment où les hypothèses alarmistes émises aux Etats-Unis dans les années 60, sur l'évolution de la population mondiale, et le rapport du Club de Rome, qui a soulevé la question de l'épuisement des ressources, font encore des remous. Deux grandes tendances s'affrontent donc à Bucarest. D'un côté, les pays occidentaux, qui déclarent que la croissance démographique des pays du Tiers-Monde est la cause de leur sousdéveloppement, et leur recommandent par conséquent de la limiter. De l'autre côté, la plupart des pays en développement (ceux de l'Afrique, en particulier) considèrent la croissance démographique (dont ils se réjouissent parfois) comme une conséquence du sous-développement auquel il convient de remédier.

Malgré ces divergences, un Plan d'Action (PAM) sur la population fut adopté à la presque unanimité. Il fut bien sûr remanié. Les appréciations trop malthusiennes en furent gommées, et des concessions accordées aux pays du Tiers-Monde. Le PAM reconnaît en effet que : "les politiques démographiques sont des éléments constitutifs des politiques de développement et ne sauraient en aucun cas en tenir lieu..."12.

Que la démographie soit la cause (selon certains) ou la conséquence (selon d'autres) du sous-développement, le Plan d'Action Mondial de Bucarest a tout de même eu le mérite, de souligner l'interdépendance des variables démographiques et économiques et sociales, et donc la nécessité d'intégrer la population dans les plans de développement, ouvrant ainsi la voie à la formulation et à la mise en 
oeuvre des nolitiques démographiques. Celles-ci, selon le PAM, relèvent du droit souverain de chaque nation. Néanmoins, le rôle de la coopération internationale en la matière est reconnu.

Le PAM a fait un certain nombre de recommandations prenant en considération la situation démographique des pays, leurs objectifs nationaux et les valeurs nationales. Par exemple (recommandation 17): "les pays qui considèrent que leur taux de croissance démographique actuel ou celui qu'ils prévoient fait obstacle à la réalisation de leurs objectifs en matière de bien-être social, sont invités à envisager d'adopter, s'ils ne l'ont pas déjà fait, des politiques démographiques entrant dans le cadre du développement socio-économique et compatibles avec les droits fondamentaux de la personne humaine...".

Les pays voulant maîtriser leur croissance démographique, ne devraient toutefois pas imposer des mesures autoritaires. En effet, le PAM recommande à tous les pays (recommandation 29):

- de respecter et d'assurer, indépendamment de leurs objectifs démographiques d'ensemble, le droit des personnes à décider librement, en connaissance de cause, et en toute responsabilité, du nombre de leurs enfants et de l'espacement des naissances;

de favoriser une éducation appropriée sur la parenté responsable, et de mettre à la disposition des personnes qui le souhaitent des services d'information à ce sujet et des moyens de l'exercer.

La planification familiale qui doit être incluse dans le programme général de santé, doit non seulement aider à espacer les naissances, mais elle doit aussi avoir pour but de prévenir les grossesses non désirées et de lutter contre la stérilité involontaire, de façon que tous les couples soient à même d'avoir le nombre d'enfants qu'ils désirent. Elle doit être incluse dans le programme général de santé.

Pour qu'une politique de population soit formulée et mise en oeuvre, le PAM recommande aux pays d'améliorer leurs connaissances démographiques, par le biais de programmes de recensement (tous les 
pays devaient avoir effectué des recensements de population entre 1975 et 1985), des statistiques d'état civil et enfin par diverses enquêtes démographiques. L'intégration des variables démographiques dans les plans de développement économique et social, autre étape de la formulation de la politique de population, devait être confiée à un service spécialisé à créer.

La conférence mondiale sur la population de Bucarest, est-elle pour quelque chose, dans les changements envers les problèmes démographiques, observés en Afrique en général et au Cameroun en particulier entre 1974 et 1984 ? Si cette conférence n'est pas directement responsable de la décision exprimée en 1980 par le gouvernement camerounais, quant à la maîtrise de la croissance de la population, il est tout de même vrai qu'elle a aidé le Cameroun et les autres pays de l'Afrique sub-saharienne, à prendre conscience des relations d'interdépendance entre population et développement.

Mais ce rôle de "transmission" de l'expérience en matière de politique de population ${ }^{13}$ ne s'arrête pas à cette prise de conscience. Les résolutions et recommandations contenues dans le PAM (lesquelles, rappelons-le, n'étaient pas obligatoires) ont été assez bien suivies par le Cameroun. D'abord au niveau de l'amélioration des connaissances démographiques; avec l'aide du FNUAP, le pays a effectué son premier recensement de population en 1976 (mais le décret concernant ce dernier a été voté en 1973). Par la suite, le pays a réalisé en 1978 son ENF (Enquête nationale de fécondité) qui s'inscrivait dans le cadre du programme de l'EMF (Enquête mondiale de fécondité). Le deuxième recensement général de la population a eu lieu en 1987, avec l'appui financier et technique du FNUAP.

Outre l'amélioration des données démographiques, le Cameroun a également suivi la recommandation du PAM, qui voulait que chaque pays définisse une politique nationale de population. La mise en oeuvre de celle-ci se poursuit toujours.

La Conférence de Bucarest a davantage été un facteur de déblocage, dans le changement d'orientation du gouvernement camerounais que celle de Mexico, qui réaffirme en fait l'essentiel de ce qui a été dit à Bucarest, à savoir d'une part que: "les gouvernements 
devraient d'urgence mettre à la disposition de tous ceux qui le souhaitent les informations et les moyens nécessaires pour leur permettre d'avoir le nombre d'enfants qu'ils désirent" (recommandation 712). D'autre part, en tenant compte des intercalations entre variables démographiques et facteurs de développement, il est admis à l'unanimité que "les efforts au niveau national et international doivent donner la priorité aux programmes d'action intégrant la population au développement" (recommandation 702).

Pour conclure nous pouvons dire que les conférences de Bucarest et de Mexico (la première encore plus, étant donné qu'elle a débloqué la situation là où les pays en développement, dont ceux de l'Afrique, ne considéraient pas les problèmes démographiques comme primordiaux dans leur processus de développement) ont ouvert aux pays africains dont le Cameroun, la voie vers l'expérience en matière de population, et donc vers la formation et la mise en oeuvre de politiques de population, ayant surtout pour but de contrôler leur croissance démographique.

La Conférence mondiale sur la population de Bucarest, a permis de sensibiliser les différents pays du monde, à l'ampleur des problèmes démographiques et à leurs rapports étroits au développement économique et social. A Mexico, la plupart des pays en développement ne considéraient plus le développement économique et social comme un préalable à la solution des problèmes démographiques. Ils ont conscience que dorénavant toute stratégie de développement doit nécessairement intégrer l'élément population, non pas uniquement en tant que moteur de ce développement, mais aussi en tant que facteur pouvant le freiner.

\section{Le rapport du Futures Group ${ }^{14}$}

Exemple de la coopération internationale appelée de ses voeux par le PAM de Bucarest, le Futures Group a entrepris, avec l'aide de l'Agence des Etats-Unis pour le Développement International (USAID), une série d'analyses portant sur un certain nombre de pays. Ces études, qui sont à chaque fois "offertes aux dirigeants des pays pour qu'ils les examinent", portent sur les relations entre les facteurs démographiques 
et le développement, et plus spécifiquement sur leur impact économique et social.

Partant donc du présupposé qu'un accroissement rapide de la population peut avoir ou a tout simplement une incidence négative sur le développement, le but du Futures Group est d'amener les gouvernements à prendre des mesures adéquates. Le cas du Cameroun a été analysé en 1978. Par un jeu de simulation d'hypothèses, différents secteurs de l'activité économique et sociale sont passés en revue, en relation avec des projections de la population de 1975 à 2010, 2025. Deux hypothèses sont examinées : d'une part, si la fécondité reste constante, quels seront ses effets sur la capacité du Cameroun à atteindre ses objectifs de développement économique et social ? D'autre part, quelle différence y aurait-il si le programme de développement comportait des mesures destinées à ralentir la croissance démographique ? (le Futures Group dans ce deuxième cas de figure, fixe la descendance moyenne à 3 enfants par femme en l'an 2000). Il est démontré que les conséquences seraient moins graves, voire meilleures, si la fécondité baissait. Voici donc les principales conclusions de ce rapport : si l'indice de fécondité reste constant (chiffres de 1976, soit environ 5,7 enfants en moyenne par femme),

- la population, qui était de 7,661 millions d'habitants en 1976, doublera en l'an 2000 et s'élèvera à 40 millions en 2025 ; le coefficient d'enfant à charge, déjà élevé, passerait de 80 à 100 adultes en âge de travailler, à 97 en l'an 2000 et 98 en 2025 ;

on devra consacrer une part plus importante de l'investissement national total pour maintenir le niveau des services par habitant; un grand nombre de jeunes resteront sous-employés; le produit national brut par habitant progressera lentement et le pays deviendra davantage tributaire des capitaux étrangers ;

la terre arable par habitant, qui était à l'époque de 2,1 ha (1978), tombera à moins de 1 ha en l'an 2000 et à 0,4 en 2025 , ce qui mettra en danger, selon le rapport, "l'aptitude du secteur agricole à satisfaire à la fois les besoins alimentaires pour la population et à produire pour l'exportation ; 
avec l'augmentation de la population urbaine, qui passera de 2,8 millions d'habitants en 1976 à 9,9 millions en l'an 2000 et à 28 en 2025, il sera difficile de satisfaire les besoins en logements urbains, eau, installations sanitaires et autres;

- avec l'augmentation de la population scolarisable, il sera difficile de faire face à celle des besoins en investissements scolaires ;

- $\quad$ la difficulté pour assurer une meilleure santé et une santé tout court sera accrue.

En revanche, si des mesures étaient prises tout de suite pour limiter la taille moyenne des familles à 3 enfants en l'an 2000 :

- la croissance démographique se poursuivrait, mais lentement;

- le coefficient d'enfant à charge tomberait de 80 pour 100 adultes à 60 en l'an 2000, et à 50 en 2025. Ceci augmenterait considérablement l'aptitude du pays à donner à ses enfants les soins et l'éducation nécessaires ;

- la réduction de la croissance démographique entraînerait une baisse des dépenses courantes consacrées aux services publics, ce qui, selon les auteurs, libérerait des fonds pour financer des investissements dans d'autres domaines du développement ; réduirait le montant des investissements nécessaires, pour maintenir uniquement le niveau actuel des services, ferait diminuer le sous-emploi et contribuerait à améliorer la répartition des revenus et à accroître le $\mathrm{PNB} /$ habitant ;

- le nombre de jeunes arrivant sur le marché du travail augmenterait, mais plus lentement que si la fécondité restait constante ;

- la superficie des terres arables par habitant diminuerait, mais, en 2025, elle serait deux fois plus importante que si la fécondité restait constante ;

- $\quad$ il serait plus facile d'assurer de meilleurs soins de santé à la population, si celle-ci était moins nombreuse. L'introduction de services de planification familiale constituerait à elle seule, une mesure importante dans le domaine de la santé, etc. 
Ce rapport du Futures Group malgré une présentation claire et une argumentation plutôt convaincante, pèche néanmoins par son caractère alarmiste et manichéen. Son but étant avant tout de convaincre le gouvernement, ses auteurs n'hésitent pas à recourir à des arguments simples et unilatéraux, pour appuyer leur démonstration sur l'influence des facteurs démographiques sur le développement. Ce sont donc les liens entre ces deux phénomènes, et non pas leurs intercalations, qui sont analysés. C'est à croire qu'il n'y a que les variables démographiques qui influent sur les facteurs de développement, et non pas le contraire. Or le PAM de Bucarest avait souligné que l'évolution des facteurs démographiques pouvait être aussi la conséquence du sous-développement, d'où la nécessité de les intégrer dans les politiques de développement.

Malgré ces critiques et peut-être grâce à sa présentation claire, le rapport du Futures Group a eu un impact très important au Cameroun dans la mesure où, à l'instar du PAM de Bucarest, il a permis au gouvernement de mieux mesurer les conséquences de la situation démographique, révélée aussi bien par le recensement de 1976 (d'environ $2 \%$ dans les années 60, l'accroissement démographique était estimé à 2,5 \% en 1976) que par l'ENF de 1978 (l'indice de fécondité est estimé à 6,5 enfants en moyenne par femme en 1978, contre 5,4 dans les années 60). L'influence de ce rapport apparaît, dans les termes même du discours du Président de la République au Congrès de. Bafoussam.

Tout en utilisant les chiffres et les conclusions de cette analyse, M.A. Ahidjo a critiqué son ton alarmiste, et rejeté d'emblée l'idée de fixer quantitativement et arbitrairement la taille de la famille à atteindre.

\section{La fécondité des adolescentes et ses conséquences}

On dispose de très peu de données sur la fécondité des adolescentes en Afrique sub-saharienne et au Cameroun en particulier. Cependant, une étude effectuée en 1988 par la Division de la Population des Nations-Unies et publiée dans la revue "Population Newsletter" ( ${ }^{\circ} 47$ de juin 1989) sur ce sujet, tente de mesurer le phénomène. Le taux de fécondité des adolescentes est en moyenne de 
160 à 170 naissances pour 1000 adolescentes âgées de 15 à 19 ans en Afrique au sud du Sahara (ce taux varie de 40 pour 1000 en Mauritanie à 200 pour 1000 en Côte d'Ivoire). Un certain nombre de facteurs peuvent expliquer ce taux particulièrement élevě par rapport à d'autres régions du monde ( 80 pour 1000 en Asie, 100 pour 1000 en Amérique Latine): une nuptialité ou une entrée en union précoce, les attitudes à l'égard de l'avortement (généralement interdit en Afrique noire), l'offre et la demande de contraception.

La nuptialité ou l'entrée en union est très précoce en Afrique. Au Cameroun, la moitié des femmes en âge de procréer se marient ou vivent en union entre 17 et 18 ans, et $71 \%$ contractent leur premier mariage avant leur 20ème anniversaire. Si l'âge légal du mariage est de 17 ans, bien des unions sont contractées avant.

Compte tenu de ce qui vient d'être dit, la majorité des grossesses chez les adolescentes, se produisent donc à l'intérieur du mariage ou d'autres formes d'unions. La fécondité précoce résultant d'une activité sexuelle ou d'un mariage précoces, a un certain nombre d'effets négatifs sur la santé des mères et des enfants. En effet, incomplètement constituées sur le plan biologique, les adolescentes connaissent une plus grande morbidité et mortalité maternelles que les femmes âgées de plus de 20 ans. Au Cameroun, des études effectuées à la Maternité Principale de Jalonné, entre 1973 et 1976, ont révélé un taux de mortalité maternelle de 1,47 décès pour 1000 naissances. Ce taux était supérieur de $36 \%$ chez les femmes de moins de 20 ans. Le niveau de mortalité maternelle actuelle varie de 1 à 1,3 pour 1000 naissances toujours dans cette maternité. On ne dispose pas de chiffres précis au niveau national, mais on estime que ce taux serait quatre fois supérieur pour l'ensemble du pays. Il ne comprend pas les décès par avortements ou issus de grossesses édaphiques (extra-utérines). Les principales causes de cette mortalité maternelle, enregistrées en 1982 par les docteurs Nasah et Drouin, sont les suivantes :

$\begin{array}{llll}- & \text { hémorragies } & : & 43,3 \% \text { des cas } \\ - & \text { infections } & : & 16,7 \% \\ - & \text { complications médicales } & : & 15,8 \% \\ - & \text { rupture utérine } & : & 10,0 \% \\ - & \text { pré-éclampsie et éclampsie } & : & 8,3 \% .\end{array}$


Les $5,9 \%$ des cas restants représentent des causes non déclarées ou non précisées.

Les adolescentes ne connaissent pas seulement une forte mortalité maternelle, leur taux de mortalité périnatale est aussi élevé. Celles qui ont moins de 16 ans représentent $21 \%$ des grossesses à haut risque. Les complications liées à la grossesse et à l'accouchement peuvent aussi causer la stérilité.

Les adolescentes mariées ou vivant en union ne sont pas les seules concernées par la fécondité précoce et ses conséquences, les élèves (école primaire et collège) et les étudiantes le sont aussi. L'Afrique noire et plus particulièrement le Cameroun, ne sont pas les seules régions du monde où l'activité sexuelle est précoce. Mais celle-ci pose moins de problèmes là où l'éducation sexuelle est mieux assurée, la contraception plus répandue. On estime en effet, que la prévalence de la contraception, parmi les adolescentes les plus âgées (18-19 ans) et qui ont déjà eu des rapports sexuels, varie, par exemple, de $92 \%$ au Royaume-Uni à environ $50 \%$ aux Etats-Unis. Les jeunes filles au Cameroun ne bénéficient pas de ces avantages, si l'on peut dire. L'éducation sexuelle y est quasi inexistante. N'étant pas assurée à l'école, où les enseignants pensent qu'elle n'est pas de leur ressort, elle n'est pas plus prise en charge par les parents, pour lesquels c'est un sujet tabou.

Le pourcentage de l'ensemble des Camerounaises utilisant la contraception étant déjà faible (seulement $5 \%$ actuellement), il l'est encore plus pour les adolescentes, qui souvent sont très mal informées, si ce n'est pas du tout, sur ce sujet (un certain nombre de discours négatifs sont aussi tenus sur la contraception, ce qui ne favorise pas son utilisation par les jeunes filles). En l'absence donc d'éducation sexuelle et de contraception (celle-ci n'est pas offerte aux jeunes, mais n'est pas non plus demandée par elles pour différentes raisons, entre autre l'interdiction des parents, qui y voient un encouragement à la prostitution et au vagabondage sexuel), les adolescentes qui expérimentent les rapports sexuels se retrouvent dans la majorité des cas enceintes. N'ayant pas forcément voulu cette grossesse, laquelle, dans la plupart des cas, signifie interruption des études, ces jeunes filles, qui ne se sentent pas encore prêtes à assumer les responsabilités 
qu'impliquent la maternité, n'ont qu'une seule issue, l'avortement clandestin (l'avortement reste interdit au Cameroun sauf pour des raisons thérapeutiques), d'autant que la société sanctionne sévèrement les adolescentes enceintes. L'interruption de la grossesse, souvent mal pratiquée, entraîne fréquemment le décès de la mère.

Etant donné l'interdiction qui pèse sur l'avortement, on ne dispose pas de statistiques au niveau national sur ce phénomène. Cependant, un certain nombre d'études ont essayé de mesurer son intensité dans les grandes villes, où il est essentiellement pratiqué. On estime ainsi, qu'à la Maternité Principale de Jalonné, 10 à $15 \%$ des grossesses finissent par un avortement provoqué. Toujours dans cette maternité, une étude menée en 1989 (elle n'était pas encore publiée à l'époque où nous nous sommes rendus au Cameroun : juillet-septembre 1989) par le Professeur Leke, gynécologue-obstétricien, révèle que, sur 800 patientes reçues en moyenne par mois, $30 \%$ viennent pour un problème lié à l'avortement : c'est-à-dire menace d'avortement (50\% des cas), avortement incomplet, complet, etc. La majorité de ces femmes, consultant pour un problème lié à l'interruption de grossesse, sont des jeunes filles, élèves ou étudiantes $(50 \%)$, avec une parité faible mais non nulle (60\% avaient déjà un enfant).

Ces avortements provoqués ayant nécessité une hospitalisation, l'avaient été à $60 \%$ par les jeunes filles elles-mêmes, très souvent dans de très mauvaises conditions, mettant leur vie en danger. Ce sont ces mauvaises conditions et les hémorragies qui en ont résulté qui ont conduit les femmes à l'hôpital, quand elles n'étaient pas décédées. Les quelques chiffres dont on dispose, n'indiquent donc pas la prévalence réelle du phénomène, d'autant que certains avortements peuvent réussir (donc passer inaperçus) ou provoquer le décès de la jeune fille en dehors de l'hôpital, donc ne pas être répertoriés.

Si les jeunes filles recourent à l'avortement, c'est pour se débarrasser d'une grossesse qu'elles n'ont pas souhaitée. Mais il est difficile de mesurer l'ampleur des grossesses non désirées dans la mesure où les femmes qui n'ont pas souhaité une grossesse n'avortent pas forcément. Cela dit, étant donné que la plupart des femmes qui viennent à l'hôpital, pour un problème lié à l'avortement sont en majorité des jeunes élèves ou étudiantes, on pourrait conclure que les 
cas d'avortement constatés chez ces jeunes femmes, sont une indication des grossesses non désirées, même s'il est vrai que certaines de ces adolescentes, résignées ou ne pouvant avorter (par crainte des risques que cela représente) décident de garder leur grossesse. Les grossesses non voulues débouchent aussi parfois sur des abandons d'enfants et, cas plus tragiques, sur l'infanticide.

Tout ce qui précède souligne la nécessité d'une éducation sexuelle, et de programmes de planification familiale, qui permettent de prévenir les grossesses non désirées, donc les avortements clandestins, et qui doivent également permettre de prévenir les maladies sexuellement transmissibles, très souvent cause de stérilité, quand elles ne sont pas soignées ou le sont mal. Il ressort d'ailleurs d'une étude menée auprès d'écolières des zones urbaines et rurales, qu'environ $20 \%$ souffraient de blennorragie (étude mentionnée par le Pr. Nasah dans sa communication au Colloque sur le Bien-être familial tenu à Jalonné du 4 au 6 août 1987).

L'activité sexuelle ne pouvant être interdite aux adolescentes, il s'avère nécessaire de les informer, de les sensibiliser à la contraception, et de leur en faciliter l'accès. Mais ceci est rendu difficile par l'insuffisance des services de planification familiale (lesquels, lorsqu'ils existent sont très peu, voire pas du tout orientés vers les jeunes) et par le fait que ces jeunes filles, doivent, lorsqu'elles ne sont pas majeures (l'âge de la majorité est de 21 ans) demander l'autorisation parentale qui leur est souvent refusée. Il est également nécessaire de relever l'âge legal du mariage, et de favoriser l'éducation des jeunes filles, car le mariage et la fécondité précoces ne favorisent pas la scolarisation, ne facilitent pas l'instruction des femmes, n'améliorent pas leur statut socio-économique, et ne facilitent pas par conséquent, la maîtrise et la planification de leur existence.

Après avoir étudié les effets moteurs du changement, voyons maintenant ses effets induits. 


\section{Les effets induits : les actions entreprises depuis $\mathbf{1 9 8 0}$}

La volonté du gouvernement camerounais de ralentir l'accroissement démographique du pays s'est traduite dans les faits par la modification de l'ancienne législation sur la contraception. En effet, la loi ${ }^{\circ} 69 / 69$ du 29 mai 1969, qui interdisait la vente et l'information sur les contraceptifs, fut abrogée en 1980 (loi $n^{\circ} 80 / 10$ du 14 juillet 1980). Les Camerounais peuvent donc depuis cette date, se procurer librement des contraceptifs aux oestrogènes sur ordonnance médicale.

Si la législation concernant la contraception a été modifiée, en revanche celle qui interdisait l'avortement, à l'exception de l'avortement thérapeutique, est restée intacte. La politique des revenus du gouvernement (système de prestations familiales et d'imposition) n'a pas subi non plus de modification, et rien pour l'instant n'indique qu'il en sera autrement.

Une autre mesure va dans le sens des nouvelles orientations gouvernementales : la création le 4 février 1984 du Ministère de la Condition Féminine. D'abord relié au Ministrère des Affaires Sociales, ce Ministère est actuellement autonome. La femme (et l'amélioration de sa condition) étant au centre de la mission attribuée au Ministère de la Condition Féminine, on comprend aisément le rôle que peut jouer ce dernier dans la "responsibilisation", la sensibilisation et l'éducation aux problèmes démographiques.

Hormis ces quelques mesures, différents projets et programmes ont été mis sur pied qui visent à traduire dans la pratique, le désir du gouvernement camerounais de maîtriser la croissance de la population. La réalisation d'un de ces projets et peut-être le plus important, fut la création par décret $n^{\circ} 85 / 275$ du ler mars 1985 de la Commission Nationale de la Population (CNP). Cette institution a pour objectif principal d'assister le gouvernement dans la définition, l'orientation et l'harmonisation de sa politique de population en matière de fécondité, et dans la recherche des voies et moyens nécessaires à sa mise en oeuvre. La CNP a tenu sa première session le 6 novembre 1985. Au cours de celle-ci, 137 résolutions dont 9 ont particulièrement trait à la fécondité (résolutions 105 à 113) ont été adoptées. 
Dans son objectif d'élaborer une politique de fécondité basée sur la parenté responsable, la CNP est secondée par une organisation exécutrice qui lui sert également de secrétariat permanent : l'Unité de Planification de la Population (UPP), créée en 1984, par un accord signé entre le gouvernement camerounais et le FNUAP. Basée au sein de la sous-direction de la planification du Ministère du Plan et de l'Aménagement du Territoire, l'UPP a pour rôle principal de mener et de coordonner les études nécessaires à la formulation et à la mise en oeuvre de la politique de population. Parmi ses autres objectifs, cette cellule est chargée de l'intégration des variables démographiques, dans les plans de développement. Cette dernière est devenue effective dès le Vème plan (1981-1986) qui aborde de façon explicite le comportement démographique.

L'UPP a organisé dès 1985 trois séminaires nationaux sur "Population, Ressources et Développement". Les résultats de ce séminaire ont été pris en considération par la CNP, pour la réalisation de sa première session.

Le Gouvernement a également prévu, un programme de sensibilisation et d'éducation de la population, aux questions démographiques. L'objectif de ce programme est de préparer la population, à prendre conscience de la nécessité de contrôler la fécondité, et de disposer de services efficaces pour la mise en oeuvre d'un programme de planification familiale. Plusieurs projets (que nous citons brièvement) doivent répondre à cet objectif clé, à savoir :

1. Le projet "Education des populations à la parenté responsable et amélioration des conditions de vie familiale et communautaire". Démarré en avril 1985 et mené par le Ministère des Affaires sociales, ce projet ne portait dans un premier temps que sur quelques foyers expérimentaux. Cette expérience devait s'étendre à l'ensemble du pays dans une seconde phase (août 1988-décembre 1990) au cours de laquelle a été réalisée l'enquête sur la famille camerounaise (1987).

2. Le projet "Femmes et développement : formation des femmes par les réseaux des maisons de la femme". Démarré en juillet 1988 et mené par le Ministère de la Condition féminine, ce 
projet se donnait pour but, de contribuer à la mise en oeuvre des objectifs du VIème plan quinquennal (1986-1991) dans le domaine de l'éducation, de la formation et de l'amélioration des conditions de vie de la femme camerounaise.

3. Le projet "Information, éducation êt communication en matière de population dans les milieux scolaires", exécuté par le Ministère de l'Education nationale. Ce projet a pour but d'utiliser l'école, comme relais permettant de mieux toucher les jeunes, et de les sensibiliser aussi bien aux problèmes démographiques, qu'à l'éducation sexuelle encore quasi inexistante dans les programmes d'enseignement.

L'entreprise est aussi un des cadres où l'on peut sensibiliser la population aux questions démographiques.

4. Le projet "Education à la vie familiale pour les travailleurs organisés", exécuté par le Ministère du Travail et de la Prévoyance sociale, avec le concours de l'OSTC (Organisation du Syndicat des Travailleurs Camerounais) et du FNUAP, entend favoriser la collaboration entre le gouvernement et les partenaires privés, en vue d'améliorer les conditions de vie des travailleurs et de leurs familles, en organisant des programmes éducatifs relatifs à la famille, à la parenté responsable et à l'amélioration de la santé de la mère et de l'enfant. Démarré en juillet 1988 , ce projet devait durer trois ans. Au cours de cette période, une enquête socio-démographique sur la vie familiale des travailleurs de l'OSTC était prévue, et devait être réalisée avec le concours de l'IFORD et de la direction des études et enquêtes du Ministère du Travail.

5. Le projet "Information, éducation et communication en matière de population" a pour but principal de contribucr, avec l'aide des grands médias (radio, télévision, presse) et l'utilisation des canaux de communication populaire (saynètes, proverbes, chansons, théâtre, etc.) à la sensibilisation de toutes les couches de la population aux problèmes liés à la politique nationale de la population. D'une durée de trois ans (démarrage en juillet 1988), le projet "IEC" comprenait également la sensibilisation 
des hommes de média aux questions démographiques. Des sěminaires étaient prévus à leur intention (dont le Séminaire de sensibilisation des journalistes africains aux questions de population, Yaoundé, 10-12 mai 1989) de même que l'introduction de l'enseignement de la démographie dans les écoles de journalisme dès l'année académique 1988/1989.

6. Les projets "Développement et bien-être familial" et "Offre des services de planification familiale" exécutés au sein du Ministěre de la Santé avec l'assistance technique de l'OMS.

Outre ces projets, qui impliquent la plupart des cellules de base de la société (écoles, entreprises, maisons de la femme, etc.), et les réseaux de communication, le gouvernement camerounais a prévu dans la mise en oeuvre de sa politique de population en matière de fécondité, d'autres programmes qui touchent de près ou de loin à celle-ci, dont un programme de recherche sur les questions démographiques. Ce dernier mené par l'Unité pour la Planification de la Population, doit permettre non seulement le suivi et l'évaluation de la mise en oeuvre de la politique démographique, mais également améliorer le niveau général des connaissances démographiques. Il est prévu à cet égard d'améliorer, voire installer là où il n'existe pas encore, un système permanent d'état civil.

Pour clore ce chapitre, il est important de signaler que malgré une profusion de projets et programmes, peu de mesures concrètes ont suivi les changements des discours gouvernementaux. Ainsi s'il est vrai que l'interdiction qui frappait l'information et la vente des contraceptifs a été levée, on constate que cette mesure demeure insuffisante. En effet l'accès à la planification familiale reste difficile. Il est toujours exigé aux femmes mariées l'autorisation de leurs maris pour pouvoir bénéficier des services de planification familiale. Il en est de même des jeunes filles, qui doivent avoir le consentement de leurs parents.

Une Commission nationale de la population a été créée, mais la plupart des résolutions qui ont été adoptées lors de sa première session, ne se sont pas encore muées en mesures effectives et réglementaires. En somme, la politique de population qui devait être formulée par la CNP, si elle est en train d'être mise en oeuvre, n'est pas encore 
adoptée, et la période actuelle de bouleversements sociaux, économiques et politiques que traverse le Cameroun, ne permet pas de prévoir une avancée réelle dans ce sens.

\section{Conclusion}

Comme dans la plupart des pays d'Afrique au Sud du Sahara, on assiste au Cameroun, à un bouleversement des positions gouvernementales, quant à l'évolution démographique. Longtemps considéré comme un facteur ayant un effet positif sur le développement économique du pays, en ce sens qu'en amont, en tant que moyen de production, elle pouvait pallier à l'insuffisance des capitaux, et qu'en aval, elle ouvrait des perspectives pour l'écoulement de la production, la croissance démographique a longtemps été encouragée par les autorités. Celles-ci y voyaient non seulement une nécessité économique, mais aussi une nécessité politique, dans la mesure où une forte population était synonyme de puissance et de grandeur nationales.

Considéré hier comme une solution au développement, le facteur "population" est pourtant devenu pour les autorités camerounaises, un obstacle à ce même développement, lequel risque de se voir freiné, voire arrêté, si aucune mesure n'est prise pour contrôler les naissances. La transition "politico-démographique" observée au Cameroun, c'est à dire, comme le dit Patrick Gubry, le passage du natalisme à l'anti-natalisme, s'est faite progressivement entre 1974 et 1984, autrement dit entre les deux Conférences mondiales sur la population (Bucarest en 1974 et Mexico en 1984). Le fait que cette transition ait eu lieu au cours de cette période, nous a amenés à nous poser la question: du rôle spécifique de la Conférence de Bucarest, sur le changement de position du gouvernement camerounais. Cette conférence a en effet, comme nous l'avons souligné, aidé le Cameroun, mais aussi la plupart des pays en développement, à prendre conscience de l'interdépendance entre la population et le développement économique et social.

Les influences extérieures n'ont pas été les seules responsables de la transition "politico-démographique" observée. D'autres facteurs que nous avons également mentionnés, l'ont aussi à des degrés divers 
provoquée. Ce changement, selon nous, était nécessaire, voire inévitable. Une meilleure connaissance de la population, grâce aux recensements et aux enquêtes démographiques réalisés entre 1974 et 1984, a en effet révélé que la population croissait à un rythme supérieur aux' estimations prévues par les plans quinquennaux de développement. Si cette croissance $(2,5 \%$ en 1976) est restée, tout au long des années 70 en deça de la croissance économique (le taux de 7,5 \% par an fut même enregistré au milieu des années 70), ce n'est plus le cas aujourd'hui où l'évolution démographique, assez impressionnante, que connaît le Cameroun (2,92 \% en 1987, soit un temps de doublement de 24 ans de la population), risque de faire obstacle au développement économique et social.

L'évolution des idées et des faits ainsi constatée va certainement se renforcer au cours des prochaines années, en raison non seulement des difficultés économiques internes, mais aussi du contexte international. C'est dans cette perspective qu'il convient de situer les actions actuellement menées par le gouvernement pour la mise en place effective d'une politique nationale de population. 


\section{ANNEXE}

Tableau 1 : Situation démographique du Cameroun : Evolution des principaux indices

\begin{tabular}{|l|r|r|r|r|}
\hline \multicolumn{5}{|c|}{ Périodes et années } \\
\hline Indice & $1960-1965$ & 1976 & 1978 & 1987 \\
& (a) & (b) & (c) & (d) \\
\hline $\mathrm{TBN}^{0} / 00$ & 40,1 & 45,0 & - & 41,2 \\
$\mathrm{SNR}^{\mathrm{T}}$ & 5,4 & 6,4 & 6,5 & 5,9 \\
$\mathrm{TBM}^{0} / 00$ & 25,0 & 20,3 & 20,2 & 14,0 \\
$\mathrm{TMI}^{0} / 00$ & 172,0 & 156,5 & 113,0 & 91,9 \\
EO(années) & 37,5 & 44,4 & 45 & 53 \\
r\% & 1,5 & 2,47 & - & 2,92 \\
\hline
\end{tabular}

$\mathbf{r}=$ taux d'accroissement naturel

Sources : $\quad$ (a) Enquête par sondage de 1960-1965

(b) Recensement général de la population de 1976

(c) Enquête nationale de la population de 1978

(d) Recensement général de la population de 1987

Tableau 2 : Pourcentage de femmes jamais enceintes ou sans grossesse féconde (femmes en union depuis plus de 5 ans) au Cameroun en 1978 selon différents groupes d'âge

\begin{tabular}{|l|r|c|}
\hline Age actuel & $\begin{array}{c}\text { \% jamais } \\
\text { enceintes }\end{array}$ & $\begin{array}{c}\text { \% sans grossesse } \\
\text { féconde }\end{array}$ \\
\hline $15-19$ & 10 & 12 \\
$20-24$ & 10 & 11 \\
$25-29$ & 9 & 11 \\
$30-34$ & 9 & 9 \\
$35-39$ & 9 & 11 \\
$40-44$ & 10 & 11 \\
$45-49$ & 11 & 14 \\
$50-54$ & 24 & 28 \\
& & 12 \\
\hline
\end{tabular}

Source: $\quad$ ENF, Rapport principal, Vol. I, Analyse des principaux résultats. Tableaux, 5.9, 1978. 
Tableau 3 : Pourcentage de femmes en union depuis plus de 5 ans sans grossesse féconde selon la région de résidence

\begin{tabular}{|c|c|c|c|c|c|c|c|c|c|}
\hline Cameroun & C-S & E & Lìt & N & N-O & O & S-O & Ydé & Dla \\
\hline 12 & 16 & 12 & 8 & 18 & 5 & 5 & 3 & 11 & 6 \\
\hline
\end{tabular}

Source: $\quad$ ENF, Rapport principal, vol. I, tableaux 5.32 et 5.33, 1978.

C-S : Centre-Sud

$\mathrm{N}:$ Nord

S-O : Sud Ouest
E : Est

$\mathrm{N}-\mathrm{O}:$ Nord Ouest

Ydé : Jalonné
Litt.: Littoral

$\mathrm{O}:$ Ouest

Dla : Douala

Tableau 4 : Sommes des naissances réduites selon la région de résidence

\begin{tabular}{|c|c|c|c|c|c|c|c|c|c|}
\hline Cameroun & C-S & E & Lit. & N & N-O & O & S-O & Ydé & Dla \\
\hline 6,5 & 6,3 & 6,6 & 5,8 & 5,4 & 6,3 & 7,2 & 6,9 & 5,2 & 5,1 \\
\hline
\end{tabular}

Source: $\quad$ ENF, Rapport principal, Vol. I, tableau 5.34, 1978. 
Notes

1. Bafoussam est la ville principale de l'ouest du Cameroun

2. Stassart J. Les avantages et les inconvénients économiques d'une population stationnaire. Faculté de droit de Liège. La Haye, 1965, 256p.

3. Richard Dackam : Croissance démographique et développement socio-économique du Cameroun. Colloque national sur le bienêtre familial. Jalonné, 4-7 août 1987.

4. Chesnais J.C. : La Transition démographique : Etapes, formes. implications économiques. INED, PUF. Paris, 1986. 580p.

5. Evina Akam : "Infécondité et sous-fécondité : évaluation et recherche des facteurs. Le cas du Cameroun." Les cahiers de l'IFORD. n¹, Février 1990, p. 147.

6. Center for Disease Control Family Planning Methods and Practice: Africa, Atlanta, Georgia, 1983.

7. Yana Simon David : La situation démographique du Cameroun et la planification familiale. Mémoire de maîtrise de sociologie. Université de Jalonné, 1985.

8. Kwekem Fankam Michel. "Fécondité et développement". In Population. ressources et développement du Cameroun. MINPAT. Jalonné, 1988.

9. Kwekem Fanfam Michel, op. cit.

10. Patrick Gubry. Cameroun: d'un natalisme nuancé vers un antinatalisme modéré? CEPED, Paris, 1988.

11. Nations Unies - 1975 - Rapport de la Conférence mondiale sur la population à Bucarest, 19-30 août 1974, New York, p.7. 
12. Mpembele Sala-Diakanda : Transmission internationale en subsaharienne de l'expérience en matière de politique de population. UN/IUSSP expert group on the international transmission of population policy expérience, 27-30 Juin 1988.

13. The Futures Groups - 1978 - Cameroun. The interrelations of population and development. Washington (D.C.), 33 p. + croquis. Document publié dans le cadre du projet RAPID (Ressources for the Awareness of the Population impacts on Development).

14. Patrick Gubry. 1988. "Cameroun : d'un natalisme nuancé vers un anti-natalisme modéré ?" Les dossiers du CEPED, $n^{\circ} 2$, Paris, 29p. 


\section{Bibliographie et références}

Center for Disease Control. Family Plamning Methods and Practices: Africa. Atlanta, Georgia, 1983.

Chamie, J. "Les positions et politiques gouvernementales en matière de fécondité et de planification familiale", pp. 167-190. Population et sociétés en Afrique au Sud du Sahara. Paris, éd. l'Harmattan, 1988, $551 \mathrm{p}$.

Chesnais, J.C. La transition démographique : étapes. formes. implications économiques. Paris, INED, PUF, 1986, 580 p.

Dackam Ngatchou, R. 1987. "Croissance démographique et développement socio-économique au Cameroun" Colloque national sur le Bien-Etre familial. Jalonné (4-6 août 1987), 21 p.

Gubry, P. 1988. "Cameroun : d'un natalisme nuancé vers un antinatalisme modéré ?" CEPED, dossiers n², Paris, 1988, 25 p.

Isaacs, S.L. \& Cook, R.J. 1985 "Lois et politiques affectant la fécondité : une décennie de changements". Population Reports (Baltimore), série $\mathrm{E}, \mathrm{n}^{\circ} 7,54 \mathrm{p}$.

Jones, M.E. La mortalité et la politique sanitaire : une mesure du développement. Département de l'Information de l'ONU, Division de l'Information économique et sociale, New York, 1984, 29 p.

Kwekem Fankam, M. "Fécondité et développement", pp.102-128. In: Population. ressources et développement du Cameroun. MINPAT, Jalonné, 1988, 504 p.

Nasah, B.T. (Communication). Colloque national sur le Bien-Etre familial. Jalonné (4-6 août 1987), 31 p. 
Nasah, B.T. "Mortalité maternelle au Cameroun". Annales du Centre universitaire des sciences de la santé. Vol.4, $\mathrm{n}^{\circ} 1$, mars 1987.

Nations-Unies. 1975 Rapport de la Conférence mondiale des NationsUnies sur la Population. Bucarest (19-30 août 1974). New York, $169 \mathrm{p}$.

Nations-Unies. Rapport de la Conférence internationale sur la population. Mexico (6-14 août 1984). New York, 1984, 109 p.

Population Newsletter : "Adolescents Fertility in Less Developed Countries". Population Division, Department of International Economic and Social Affairs. United Nations Secretariat, New York, ${ }^{\circ} 47$, June 1989.

Sala-Diakanda, M. "De Bucarest à Mexico : évolution des politiques africaines en matière de population". CEPED, dossier $n^{\circ} 2$, Paris, 1988, 14 p.

Stassart, J. Les avantages et les inconvénients économiques d'une population stationnaire. Faculté de Liège, la Haye, 1965, 265 p.

The Futures Group. 1978. Cameroon. The Interrelation of Population and Development. Washington D.C., 33 p. + croquis.

Westinghouse Social Sciences International: Demographic Data for Development - 1984 Population Policy Review : Republic of Cameroon. Colombia (Md.), 16 p.

Yana, S.D. 1985. Situation démographique du Cameroun et planification familiale. Jalonné : Université, Mémoire de maîtrise de sociologie, 99 p. multig.

Cameroon Tribune (Yaoundé) : $n^{\circ} 2223$ du 10/11/81. 